\title{
Household air pollution, ultrasound measurement, fetal biometric parameters and intrauterine growth restriction
}

Anindita Dutta ${ }^{1}$, Donee Alexander ${ }^{1}$, Theodore Karrison², Oludare Morhasson-Bello ${ }^{3}$, Nathaniel Wilson", Omolola Mojisola Atalabi ${ }^{5}$, Damilola Adu ${ }^{6}$, Tope Ibigbami ${ }^{6}$, Samuel Adekunle ${ }^{6}$, Dayo Adepoju ${ }^{6}$, John Olamijulo $^{6}$, Omolola Akinwunmi ${ }^{5}$, Oluniyi S. Afolabi ${ }^{5}$, Oluwafunmilade Deji-Abiodun ${ }^{1}$, Babatunde Adedokun ${ }^{1}$, Briseis Aschebrook-Kilfoy ${ }^{2}$, Oladosu Ojengbede ${ }^{3}$ and Christopher O. Olopade ${ }^{1^{*}}$ (D)

\begin{abstract}
Background: Low birthweight, intrauterine growth restriction (IUGR) and perinatal mortality have been associated with air pollution. However, intervention studies that use ultrasound measurements to assess the effects of household air pollution (HAP) on fetal biometric parameters (FBP) are rare. We investigated the effect of a cookstove intervention on FBP and IUGR in a randomized controlled trial (RCT) cohort of HAP-exposed pregnant Nigerian women.

Methods: We recruited 324 women early in the second trimester of pregnancy. Between 16 and 18 weeks, we randomized them to either continue cooking with firewood/kerosene (control group) or receive a CleanCook stove and ethanol fuel (intervention group). We measured fetal biparietal diameter (BPD), head circumference (HC), femur length (FL), abdominal circumference (AC) and ultrasound-estimated fetal weight (U-EFW) in the second and third trimesters. The women were clinically followed up at six regular time points during their pregnancies. Once during the women's second trimester and once during the third, we made 72-h continuous measurements of their personal exposures to particulate matter having aerodynamic diameter $<2.5 \mu \mathrm{m}\left(\mathrm{PM}_{2.5}\right)$. We adopted a modified intent-to-treat approach for the analysis. Differences between the intervention and control groups on impact of HAP on fetal growth trajectories were analyzed using mixed effects regression models.
\end{abstract}

Results: There were no significant differences in fetal growth trajectories between the intervention and control groups.

Conclusions: Larger studies in a setting of low ambient air pollution are required to further investigate the effect of transitioning to a cleaner fuel such as ethanol on intrauterine growth.

Trial registration: ClinicalTrials.gov NCT02394574; September 2012

Keywords: Household air pollution, Fetal growth, Ultrasound measurement, Intrauterine growth restriction, Pregnant women, Nigeria

\footnotetext{
* Correspondence: solopade@bsd.uchicago.edu

'Department of Medicine and Center for Global Health, University of Chicago, Chicago, USA

Full list of author information is available at the end of the article
}

(C) The Author(s). 2021 Open Access This article is licensed under a Creative Commons Attribution 4.0 International License, which permits use, sharing, adaptation, distribution and reproduction in any medium or format, as long as you give appropriate credit to the original author(s) and the source, provide a link to the Creative Commons licence, and indicate if changes were made. The images or other third party material in this article are included in the article's Creative Commons licence, unless indicated otherwise in a credit line to the material. If material is not included in the article's Creative Commons licence and your intended use is not permitted by statutory regulation or exceeds the permitted use, you will need to obtain permission directly from the copyright holder. To view a copy of this licence, visit http://creativecommons.org/licenses/by/4.0/ The Creative Commons Public Domain Dedication waiver (http://creativecommons.org/publicdomain/zero/1.0/) applies to the data made available in this article, unless otherwise stated in a credit line to the data. 


\section{Background}

Early-life exposure to environmental contaminants, such as those in household air pollution (HAP), can lead to development of early childhood diseases like asthma, wheezing, respiratory infections, and altered immune defense; also, chronic diseases like diabetes and heart disease [1]. Further, maternal exposure to high levels of air pollutants during pregnancy has been associated with increased risk of adverse pregnancy outcomes such as preterm birth, stillbirth, small-for-gestational-age babies, low birth weight and neonatal death [2-5]. A major source of prenatal and early-life HAP exposure, globally, is the highly prevalent practice of using biomass, kerosene and coal for household cooking and/or heating purposes. Hence, it is necessary to assess fetal health and ultimate birth outcomes among pregnant women exposed to HAP from using biomass, kerosene and coal for cooking. An informative assessment of fetal health is continuous monitoring of fetal growth for the duration of entire pregnancies.

Ultrasound examinations result in reliable and reproducible fetal biometric measurements [6]. Ultrasound is the gold standard approach for measuring fetal growth during pregnancy [7]. The method has been used to examine the impacts of early-life exposure to air pollution on fetal growth. Most such studies have concerned the impact of exposure to ambient air pollution [2,8-11]. To date, no studies have used ultrasound serially to measure fetal biometric parameters in cook stove intervention trials.

Patelarou and Kelly [12] suggested that fetal growth should be assessed serially during pregnancy rather than only at birth and, further, that direct methods of assessment such as ultrasound provide better insight into the specific effects of maternal exposure to HAP. Therefore, we undertook this study to assess the impact of exposure to HAP in a randomized controlled trial (RCT) of a cook stove fuel intervention, using ultrasound to measure fetal growth during pregnancy. The trial took place in Ibadan, Nigeria, and investigated the impact, on pregnancy outcomes, of transitioning women from cooking with firewood or kerosene to cooking with ethanol. We performed ultrasound measurement of fetal growth six times during the pregnancy on each woman who participated in this study. We hypothesized that the fetuses of the control group women, who were exposed to higher levels of $\mathrm{PM}_{2.5}$ from use of firewood and kerosene, would exhibit statistically significant intrauterine growth restriction (IUGR), compared with the fetuses of the intervention group women, who used cleanburning ethanol fuel and, thus, were exposed to lower levels of $\mathrm{PM}_{2.5}$.

\section{Methods}

Study design, participant recruitment and eligibility criteria

In earlier publications we have detailed the processes of participant recruitment and randomization and the eligibility criteria for this RCT [13-15], which was conducted in Ibadan, Nigeria between June 2013 and October 2015. Participants were randomized using randomization module in REDCap to ethanol or control cook stove group. Among 324 participants, half were randomly assigned to control arm (firewood/kerosene), who continued to cook with the fuel they were using at entry, and the other half to ethanol arm, who received CleanCook ethanol stove (CLEANCOOK Sweden AB). Women $(n=324)$, who were apparently healthy, non-smokers and non-chewers of tobacco, cooked regularly with firewood/kerosene, and were at $<18$ weeks gestational age, were enrolled to this study. Exclusion criteria included smoking, living with smokers, cooking for a living, HIV-positive status, high-risk pregnancy (multiple gestations, uncontrolled maternal hypertension, maternal age $>35$ years for first delivery, three/more prior miscarriages, or a prior Cesarean-section).

With potential recruits, we used a portable ultrasound device to determine gestational age (GA), assembling a sample of 324 participants. Between 16 and 18 weeks fetal GA, we randomized participants either to receive a CleanCook stove and a supply of ethanol fuel (intervention arm, E) throughout pregnancy or to continue cooking with firewood or kerosene (control arm, C). We then followed all participants to term. The average duration of study participation was 156 days.

The study protocol was explained to the participants in detail and their written informed consents were obtained at the time of recruitment. The work has been carried out in accordance with the Code of Ethics of the World Medical Association (Declaration of Helsinki) and the Institutional Review Boards of the University of Chicago and the University of Ibadan approved the study protocol, which was registered as NCT02394574 on ClinicalTrials.gov.

\section{Questionnaire survey}

We gathered information on the participants' age, level of education, habits, family, dietary details, occupation of the participants, occupation of the spouse and exposure to environmental tobacco smoke (ETS), average family income, cooking hours per day, cooking-years, types of fuel used for cooking, home kitchen design (especially the presence or lack of windows), obstetrics history, current health status, pertinent past medical history and family history, through face-to-face interviews using structured questionnaires in the local language (Yoruba). 
Ultrasound measurements of fetal biometric parameters After the participating women completed the structured questionnaires and had blood drawn for routine prenatal and malaria screening, they were asked to return to the clinic the following day for the first in a series of ultrasound examinations. All women in the study registered with a clinic midwife who recorded the first day of the women's last known menstrual period (LMP), expected due date (EDD), and symphysis-fundal height (SFH). A trained radiologist from the University College Hospital (UCH) in Ibadan, Nigeria, who was unaware of the women's randomization group, performed the appropriate examination to determine estimated GA and other growth parameters in all participants, using a portable Sonosite Micromaxx ultrasound system (Bothell, WA, USA).

\section{Data collection}

We measured and recorded fetal heart rate (FHR), biparietal diameter (BPD), occipitofrontal diameter (OFD), head circumference $(\mathrm{HC})$, abdominal circumference (AC), femur length (FL), amniotic fluid index (AFI), and ultrasound-estimated fetal weight (U-EFW). Additionally, the presence of IUGR, gross fetal anomalies, uterine fibroids, gross uterine or ovarian anomalies, and placental anomalies was recorded. When possible, all measurements were taken three times at each time point and averaged. Fetal weight was estimated using the Hadlock [16] method. In addition to the day after initial clinic presentation, ultrasounds were performed at 20, 26, 30, 34 and 38-weeks GA. The variables analyzed in this report are BPD, HC, FL, AC and U-EFW.

\section{Personal exposure monitoring}

As reported in our prior publications [13-15, 17], we determined duration of cooking using a stove use temperature sensitive monitor, measured pregnant $\mathrm{Ni}$ gerian women's levels of personal exposure to $\mathrm{PM}_{2.5}$ using RTI MicroPEM for three consecutive days $(72 \mathrm{~h}$ ) at two time points: during the second and third trimesters of pregnancy. Each woman in the study carried the MicroPEM in a small, culturally appropriate bag placed near the breathing zone. An internal accelerometer, which is sensitive enough to detect breathing movement even if the woman was sitting still, served as a quality control check to ensure the monitor was worn. In our analyses, we used the mean $\mathrm{PM}_{2.5}$ across both measurement intervals. The summary parameters calculated were mean $\mathrm{PM}_{2.5}$, minimum and maximum levels of $\mathrm{PM}_{2.5}$ over $72 \mathrm{~h}$, and the time in minutes spent by the women with $\mathrm{PM}_{2.5}$ levels above $100 \mu \mathrm{g} / \mathrm{m}^{3}$. We performed PAH in a small subset of the participants and while we observed higher levels in the control group, particularly kerosene users, the numbers were too small to make any definitive and conclusive comments based on the measurements.

\section{Data analysis}

Our analysis employed the modified intent-to-treat (ITT) approach, as the final analysis excluded some participants that had no follow up data after randomization into the study groups. However, for the analysis, participants were included in the group to which they were randomized regardless of stove use compliance according to the intention to treat principle. The longitudinal fetal outcome variables analyzed were BPD, HC, FL, AC and U-EFW at time of recruitment and at each of the five subsequent time points across pregnancy: GA 20, 26, 30, 34 and 38 weeks. Analysis focused on the difference in growth patterns between the intervention (ethanol) and control (firewood/kerosene) groups. We used mixed effects models for analysis of intrauterine growth variables. For each of the growth parameters, we fitted a random coefficient model regressing the repeat measurements on intervention group in one set of analyses and on $\mathrm{PM}_{2.5}$ measurements in another. The random coefficient model treated subjects as a random effect allowing for correlation between repeat measurements of fetal growth. Additionally, the trajectories of fetal parameters (Fig. 1) indicated a non-linear trajectory as growth rate was initially high and then slowed (although the reverse was found for U-EFW), thus, a quadratic term was included for GA. The observation that some fetuses were consistently bigger than others suggested a random intercept model was indicated, while a random slope was added to the model to also allow for fetuses to differ in their overall rate of growth concerning intrauterine growth parameters.

Letting $\mathrm{y}_{\mathrm{ij}}$ denote fetal intrauterine growth measurements for subject $j$ at visit $i$, the random intercept model was:

$Y_{i j}=\beta_{1}+\beta_{2} x_{i j}+\beta_{3} x_{i j}^{2}+\beta_{4} Z_{j}+\zeta_{1 j}+\zeta_{2 j} x_{i j}+\varepsilon_{i j}[1]$.

$x_{i j}$ is the GA for subject $j$ at visit $i$.

$Z_{j}$ is a dummy variable for the intervention.

$\zeta_{1 \mathrm{j}}$ is the random intercept.

$\zeta_{2 \mathrm{j}}$ is the random slope of GA.

$\varepsilon_{\mathrm{ij}}$ is the GA specific error term that allows the growth measurements to deviate from the perfectly quadratic trajectories defined by the first four terms.

We assessed the potential importance of adding the quadratic term by examining the estimates and associated $p$-values of the square of the GA variable included in the model. In a second model, the dummy variable for the intervention was replaced with a four-category variable (quartiles) for measured $\mathrm{PM}_{2.5}$. The quartiles were determined based on the distribution of $\mathrm{PM}_{2.5}$ among controls. The random coefficient models were adjusted for mother's age, body mass index, marital 


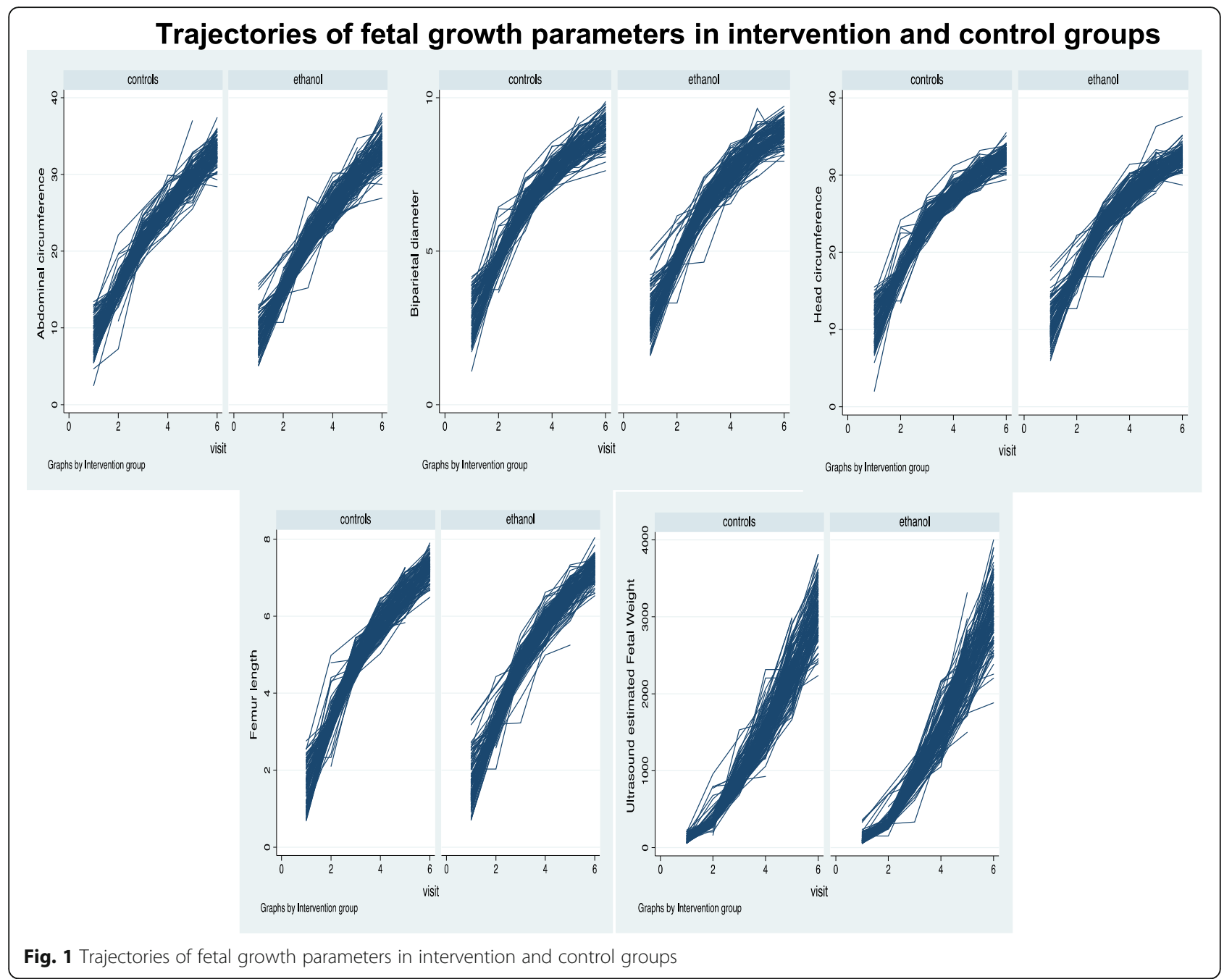

status, educational level, number of children, history of stillbirth, and history of miscarriage. Regression coefficients and 95\% confidence intervals were reported.

\section{Results}

The baseline clinical and demographic characteristics were similar between the study groups and have been reported in our previous publications [13, 14]. Overall, few women were diabetic (1.2\% ethanol-users and $1.9 \%$ firewood/kerosene users). The mean age of the mothers was 28 years (range 14-44) and mean body mass index was $24 \mathrm{~kg} / \mathrm{m}^{2}$ (range 14.2-45.0). Education levels varied from none to beyond high school. Many participants were illiterate in both groups (37.9\% ethanol users and $43.2 \%$ firewood/kerosene users). Mean GA at entry was 13 weeks, ranging from 6.7-18 weeks. We did not randomize any participant to the study's intervention or control arm until 16 weeks GA. The majority of the women had two or fewer children at enrollment. Over
$25 \%$ of them had a prior miscarriage and $8 \%$ a prior stillbirth. Of the 324 enrolled participants, 306 completed the study, while 18 participants dropped out between randomization and delivery (8 intervention; 10 control). Table 1 shows the summary data for exposure levels for the intervention (ethanol) and control (firewood/kerosene) groups.

The changes in the fetal parameters by group (intervention/control) are shown in Table 2 and Fig. 1. The growth trajectories appear similar between the two groups and there were no significant differences between the intervention and the control groups in the random effects models (Table 3 ). However, a borderline significant difference in growth parameter by quartiles of $\mathrm{PM}_{2.5}$ was found in the random effects model for U-EFW. Those in the fourth quartile of mean $\mathrm{PM}_{2.5}$ had a significantly higher mean U-EFW compared to those in the first quartile $(p=0.046)$ (Table 4, Fig. 2). 
Table 1 Summary statistics for $\mathrm{PM}_{2.5}$ levels in the study groups and overall

\begin{tabular}{|c|c|c|c|c|c|c|}
\hline \multirow[b]{2}{*}{ 2nd trimester mean 72-h $\mathrm{PM}_{2.5}$ quartile } & \multicolumn{2}{|l|}{ Intervention } & \multicolumn{2}{|l|}{ Control } & \multicolumn{2}{|l|}{ All } \\
\hline & $\begin{array}{l}\text { Median } \\
\text { (min, max) }\end{array}$ & $\mathrm{n}$ & $\begin{array}{l}\text { Median } \\
\text { (min, max) }\end{array}$ & $\mathrm{n}$ & $\begin{array}{l}\text { Median } \\
\text { (min, max) }\end{array}$ & $\mathrm{n}$ \\
\hline Q1 & $20.3(5.5-25.1)$ & 32 & $21.5(12.8-26.6)$ & 26 & $20.6(5.5-26.6)$ & 58 \\
\hline Q2 & $32.3(27-40.0)$ & 29 & $33.0(27.0-40.5)$ & 29 & $32.9(27.0-40.5)$ & 58 \\
\hline Q3 & $52.5(40.9-66.5)$ & 28 & $51.4(41.3-67.6)$ & 30 & $51.8(40.9-67.6)$ & 58 \\
\hline Q4 & $166.3(69.6-437.8)$ & 27 & $161.5(69.6-437.8)$ & 31 & $162.6(69.6-437.8)$ & 58 \\
\hline \multicolumn{7}{|l|}{ 3rd trimester mean 72-h $\mathrm{PM}_{2.5}$ quartile } \\
\hline Q1 & $26.1(0.7-32.3)$ & 28 & $22.0(8.8-31.3)$ & 24 & $23.8(0.7-32.3)$ & 52 \\
\hline Q2 & $39.7(32.7-48.2)$ & 26 & $40.9(33.9-48.9)$ & 26 & $40.1(32.7-48.9)$ & 52 \\
\hline Q3 & $62.8(49.2-106.8)$ & 27 & $71.5(49.5-113.8)$ & 25 & $64.5(49.2-113.8)$ & 52 \\
\hline Q4 & 189.8 (122.2-890.5) & 24 & $186.3(117.1-698.6)$ & 27 & 186.4 (117.0-890.5) & 51 \\
\hline
\end{tabular}

$n$ sample size; min minimum; max maximum

Table 2 Summary statistics for fetal parameters by gestational age and group (control/intervention)

\begin{tabular}{|c|c|c|c|c|c|c|c|c|c|c|}
\hline \multirow{2}{*}{ Gestational age (weeks) } & \multirow[b]{2}{*}{ BPD } & \multirow[b]{2}{*}{$\mathrm{HC}$} & \multicolumn{3}{|c|}{ Control } & \multirow[b]{2}{*}{ BPD } & \multirow[b]{2}{*}{$\mathrm{HC}$} & \multicolumn{2}{|c|}{ Intervention } & \multirow[b]{2}{*}{ U-EFW } \\
\hline & & & $\mathrm{FL}$ & $A C$ & U-EFW & & & $\mathrm{FL}$ & $A C$ & \\
\hline \multicolumn{11}{|l|}{13 weeks } \\
\hline Mean & 2.9 & 10.7 & 1.6 & 8.9 & 120.9 & 3.0 & 11.0 & 1.7 & 9.1 & 125.4 \\
\hline SD & 0.7 & 2.6 & 0.6 & 2.3 & 48.5 & 0.7 & 2.5 & 0.6 & 2.3 & 60.2 \\
\hline$n$ & 108 & 103 & 103 & 104 & 97 & 104 & 100 & 99 & 100 & 95 \\
\hline \multicolumn{11}{|l|}{20 weeks } \\
\hline Mean & 4.8 & 17.6 & 3.3 & 15.2 & 357.9 & 4.7 & 17.4 & 3.3 & 15.1 & 340.4 \\
\hline SD & 0.4 & 1.3 & 0.3 & 1.4 & 97.6 & 0.3 & 1.0 & 0.3 & 1.1 & 57.4 \\
\hline$n$ & 154 & 154 & 154 & 154 & 150 & 147 & 147 & 147 & 147 & 147 \\
\hline \multicolumn{11}{|l|}{26 weeks } \\
\hline Mean & 6.5 & 24.0 & 4.9 & 21.6 & 911.2 & 6.5 & 23.8 & 4.8 & 21.5 & 887.3 \\
\hline SD & 0.3 & 1.1 & 0.2 & 1.2 & 135.4 & 0.3 & 1.1 & 0.3 & 1.3 & 120.0 \\
\hline$n$ & 145 & 145 & 145 & 145 & 143 & 148 & 148 & 148 & 148 & 145 \\
\hline \multicolumn{11}{|l|}{30 weeks } \\
\hline Mean & 7.6 & 27.7 & 5.8 & 25.9 & 1525.0 & 7.5 & 27.7 & 5.8 & 25.9 & 1529.2 \\
\hline SD & 0.3 & 0.9 & 0.3 & 1.2 & 203.5 & 0.4 & 1.2 & 0.3 & 1.5 & 223.5 \\
\hline$n$ & 145 & 145 & 145 & 145 & 144 & 149 & 149 & 149 & 149 & 148 \\
\hline \multicolumn{11}{|l|}{34 weeks } \\
\hline Mean & 8.4 & 30.5 & 6.6 & 29.8 & 2295.6 & 8.4 & 30.5 & 6.6 & 29.9 & 2315.1 \\
\hline SD & 0.3 & 1.0 & 0.3 & 1.6 & 277.0 & 0.4 & 1.2 & 0.3 & 1.5 & 288.2 \\
\hline$n$ & 140 & 140 & 139 & 140 & 140 & 150 & 150 & 150 & 150 & 148 \\
\hline \multicolumn{11}{|l|}{38 weeks } \\
\hline Mean & 8.9 & 32.4 & 7.3 & 33.1 & 3061.7 & 8.9 & 32.4 & 7.2 & 33.1 & 3042.7 \\
\hline SD & 0.4 & 1.1 & 0.3 & 1.6 & 324.3 & 0.4 & 1.2 & 0.3 & 1.9 & 374.2 \\
\hline$n$ & 113 & 113 & 113 & 113 & 112 & 120 & 120 & 120 & 120 & 116 \\
\hline
\end{tabular}

$n$ number; SD standard deviation; $B P D$ biparietal diameter; $H C$ head circumference; $F L$ fetal length; $A C$ abdominal circumference; $U$-EFW ultrasound-estimated fetal weight 
Table 3 Regression of fetal parameters on group random intercept model*

\begin{tabular}{|c|c|c|c|}
\hline Fetal parameters & Coefficient & 95\% Confidence Interval & $p$-value \\
\hline \multicolumn{4}{|l|}{ BPD } \\
\hline Randomization group & -0.010 & -0.066 to 0.046 & 0.736 \\
\hline Gestational age (weeks) & 0.390 & 0.375 to 0.405 & $<0.001$ \\
\hline Gestational age ${ }^{2}$ & -0.003 & -0.003 to -0.002 & $<0.001$ \\
\hline \multicolumn{4}{|l|}{$\mathrm{HC}$} \\
\hline Randomization group & 0.008 & -0.185 to 0.169 & 0.926 \\
\hline Gestational age (weeks) & 1.504 & 1.450 to 1.557 & $<0.001$ \\
\hline Gestational age ${ }^{2}$ & -0.012 & -0.013 to -0.011 & $<0.001$ \\
\hline \multicolumn{4}{|l|}{$\mathrm{FL}$} \\
\hline Randomization group & -0.013 & -0.060 to 0.034 & 0.586 \\
\hline Gestational age (weeks) & 0.313 & 0.301 to 0.325 & $<0.001$ \\
\hline Gestational age ${ }^{2}$ & -0.002 & -0.002 to -0.001 & $<0.001$ \\
\hline \multicolumn{4}{|l|}{$A C$} \\
\hline Randomization group & 0.027 & -0.199 to 0.253 & 0.817 \\
\hline Gestational age (weeks) & 0.988 & 0.933 to 1.044 & $<0.001$ \\
\hline Gestational age ${ }^{2}$ & 0.0002 & -0.001 to 0.001 & 0.747 \\
\hline \multicolumn{4}{|l|}{ U-EFW } \\
\hline Randomization group & -5.336 & -27.589 to 16.917 & 0.638 \\
\hline Gestational age (weeks) & -120.744 & -127.529 to -113.959 & $<0.001$ \\
\hline Gestational age ${ }^{2}$ & 4.738 & 4.610 to 4.866 & $<0.001$ \\
\hline
\end{tabular}

SE standard error; $B P D$ biparietal diameter; $H C$ head circumference; $F L$ fetal length; $A C$ abdominal circumference; $U$-EFW ultrasound-estimated fetal weight *Adjusted for mother's age, body mass index, marital status, educational level, number of children, history of stillbirth, and history of miscarriage

\section{Discussion}

To the best of our knowledge, this study is the first randomized controlled, ethanol fuel cookstove intervention conducted with the intent of characterizing the effect of HAP exposure reduction on birth outcomes and monitoring personal exposures to $\mathrm{PM}_{2.5}$ during pregnancy in the context of an ethanol cook stove intervention. Additionally, it is the first to have used ultrasonography to make repeated (six) periodic measurements of fetal biometric parameters, including $\mathrm{BPD}, \mathrm{HC}, \mathrm{FL}, \mathrm{AC}$ and $\mathrm{U}-$ EFW in a HAP intervention study cohort. No significant differences in growth trajectories between the intervention and control groups were observed in the random effects models. Additionally, there were no significant associations between $\mathrm{PM}_{2.5}$ levels and fetal parameters, although a borderline significantly higher U-EFW at the highest quartile was in the unexpected direction, suggesting this could have been a spurious finding. We note that the study area-Ibadan, Nigeria-has relatively high ambient air pollution levels and infer that this may have played a role in minimizing any effects of the ethanol stove intervention. However, we advocate that serial ultrasound monitoring of FBP may help to identify IUGR earlier during pregnancy for women exposed to HAP.
Exposure to HAP derived from the use of biomass and kerosene for cooking has been linked to many health problems. Women and children in low-income countries disproportionately suffer from the consequences of HAP exposure, as they are most often tasked with meal preparation. In urban and periurban areas of Nigeria where this study was conducted, households predominantly utilize biomass (wood) and kerosene for cooking, with the proportion using kerosene as high as $55 \%$. This is because cleaner burning fuels such as propane are expensive and so not affordable.

Many studies have suggested a link between HAP exposure in pregnant women and the frequency of stillbirth, pre-term delivery, and low-birth weight $[2-4,18]$. These studies showed that mean birth weight is lower in households utilizing biomass fuels for cooking and even lower in households utilizing kerosene. However, most of these studies were cross-sectional in design and relied on survey instruments such as census or community health surveys. A few measured pollution levels in the kitchen but did not perform personal exposure monitoring in the pregnant women. Further, these studies are plagued by the residual confounding inherent in any cross-sectional design. 
Table 4 Regression of fetal parameters on $\mathrm{PM}_{2.5}$ exposure levels*

\begin{tabular}{|c|c|c|c|}
\hline Fetal parameters & Coefficient & 95\% Confidence Interval & $p$-value \\
\hline \multicolumn{4}{|l|}{ BPD } \\
\hline \multicolumn{4}{|c|}{ Mean $\mathrm{PM}_{2.5}$ quartile* } \\
\hline Q2 & -0.001 & -0.098 to 0.096 & 0.985 \\
\hline Q3 & 0.040 & -0.058 to 0.138 & 0.424 \\
\hline Q4 & 0.036 & -0.063 to 0.135 & 0.474 \\
\hline \multicolumn{4}{|l|}{$\mathrm{HC}$} \\
\hline \multicolumn{4}{|c|}{ Mean $\mathrm{PM}_{2.5}$ quartile } \\
\hline Q2 & -0.065 & -0.394 to 0.264 & 0.698 \\
\hline Q3 & 0.021 & -0.311 to 0.353 & 0.901 \\
\hline Q4 & 0.102 & -0.232 to 0.435 & 0.551 \\
\hline \multicolumn{4}{|l|}{$\mathrm{FL}$} \\
\hline \multicolumn{4}{|c|}{ Mean $\mathrm{PM}_{2.5}$ quartile } \\
\hline Q2 & -0.057 & -0.140 to 0.024 & 0.168 \\
\hline Q3 & -0.012 & -0.093 to 0.070 & 0.780 \\
\hline Q4 & 0.007 & -0.075 to 0.089 & 0.869 \\
\hline \multicolumn{4}{|l|}{$A C$} \\
\hline \multicolumn{4}{|c|}{ Mean $\mathrm{PM}_{2.5}$ quartile } \\
\hline Q2 & -0.216 & -0.583 to 0.151 & 0.248 \\
\hline Q3 & 0.063 & -0.306 to 0.432 & 0.738 \\
\hline Q4 & 0.083 & -0.291 to 0.457 & 0.663 \\
\hline \multicolumn{4}{|l|}{ U-EFW } \\
\hline \multicolumn{4}{|c|}{ Mean $\mathrm{PM}_{2.5}$ quartile } \\
\hline Q2 & -1.02 & -31.144 to 29.112 & 0.947 \\
\hline Q3 & 14.13 & -16.749 to 45.015 & 0.370 \\
\hline Q4 & 32.59 & 0.559 to 64.628 & 0.046 \\
\hline
\end{tabular}

$B P D$ biparietal diameter; $H C$ head circumference; $F L$ fetal length; $A C$ abdominal circumference; U-EFW ultrasound-estimated fetal weight; Reference category for $\mathrm{PM}_{2.5}$ level is the first quartile; square of gestational age was omitted from the model; * *eference category - first quartile

*Adjusted for mother's age, body mass index, marital status, educational level, number of children, history of stillbirth, and history of miscarriage

While associations between HAP exposure and adverse pregnancy outcomes have been reported, there has been little serial monitoring of IUGR using ultrasound. Thus, there is a paucity of data relating fetal growth patterns to in-utero personal exposure to HAP. Our study, which transitioned pregnant women from using kerosene and firewood to a cleaner fuel, ethanol, fills this gap by demonstrating that HAP exposure from cooking with dirty fuels is associated with adverse pregnancy outcomes [14], though the intervention did not appear to have an effect on intrauterine fetal growth.

Although our study did not find a significant effect of the intervention on fetal growth, we recommend that for future randomized controlled studies that involve an intervention to reduce pregnant women's exposures to HAP, specifically, it may be necessary to select locations where population density is lower, ambient air pollution levels are lower, and homes are separated by larger distances. This would reduce the potential for ambient air pollution and HAP from nearby houses to influence exposure level values observed from personal monitoring of individual women in their homes.

Performing ultrasound measurements at the time of recruitment and repeating these measures at GA 20, 26, 30,34 and 38-weeks during the entire pregnancy, personal monitoring of HAP exposure twice during pregnancy, and using robust statistical measures are the strengths of this study. However, there are some limitations. First, in Nigeria, women generally don't present for antenatal care until late in the second trimester. For this reason, we were unable to determine fetal growth rate during the first trimester. Fetal growth was not determined until participants were randomized during their second trimesters, between 16 and 18 weeks GA. Second, as mentioned earlier, relatively high background ambient air pollution levels in Ibadan may have minimized any beneficial effects of the ethanol stove intervention on fetal development.

Despite the above-mentioned limitations, this study stands out as the first one to utilize a sensitive tool like ultrasonography to measure the early effects of HAP exposure reduction on fetal biometric measures in a cohort of Nigerian women. Our study makes a significant contribution to the literature on the health implications of HAP exposure for pregnant women.

Assessing in-utero growth deficits due to exposure to environmental pollutants at different time points during pregnancy is of great importance from the scientific and public health perspectives. Substantial reduction in inutero exposure to HAP, to levels below the standard limits set by the World Health Organization (WHO) is important in order to promote healthy fetal growth. However, our ethanol fuel intervention was not significantly associated with better fetal growth outcomes than were observed in the control group (kerosene/firewood fuel). Larger studies with Tier 4 cooking stoves/fuels are required to augment our findings related to the health effects of HAP exposure reduction. Robust results obtained from larger studies could drive policy and business decisions in countries dealing with the adverse effects of air pollution.

\section{Conclusions}

Our study demonstrated that fetal growth parameters were not significantly different between women randomized to an ethanol cookstove intervention and those that continued to use kerosene or firewood. 


\section{Trajectories in ultrasound-estimated fetal weight by quartiles of $\mathrm{PM}_{2.5}$}

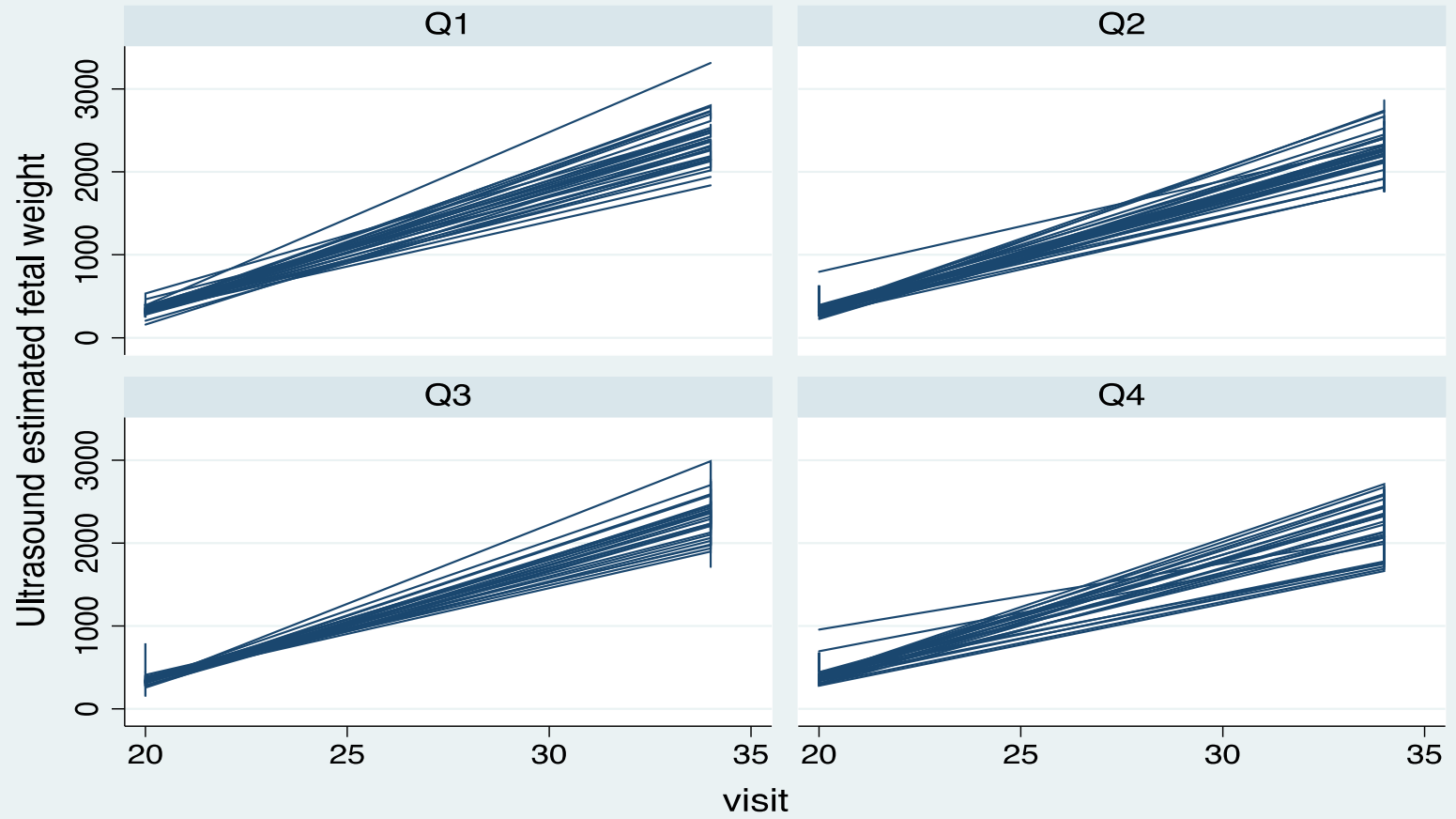

Graphs by 4 quantiles of mean72hrPM25

Fig. 2 Trajectories in ultrasound-estimated fetal weight by quartiles of $\mathrm{PM}_{2.5}$

\section{Abbreviations}

GA: Gestational age; HAP: Household air pollution; RCT: Randomized controlled trial; E: Ethanol; C: Control; FHR: Fetal heart rate; BPD: Biparietal diameter; OFD: Occipitofrontal diameter; HC: Head circumference;

AC: Abdominal circumference; FL: Femur length; AFI: Amniotic fluid index; UEFW: Ultrasound-estimated fetal weight; LMP: Last known menstrual period; EDD: Expected due date; SFH: Symphysis-fundal height; UCH: University college hospital; ITT: Intent-to-treat; HPCS: Haifa pregnancy cohort study; WHO: World health organization

\section{Acknowledgements}

The randomized control trial was funded by the United Nations Foundation through Global Alliance for Clean Cookstoves (Grant \# UNF12-378) and supported by Richard and Susan Kiphart, Project Gaia and Shell Exploratory Company. These sponsors had no role in study design, data collection, data analysis, interpretation, or writing of the report. We acknowledge the assistance of Dr. Susan Duncan for collaboration and editorial input. The corresponding author had full access to all the data in the study and had final responsibility for the decision to submit.

\section{Authors' contributions}

AD- Data review and writing first draft of manuscript and manuscript edits; DA- Study design, data collection, manuscript review and edit; TK-Study design, data analysis, interpretation, manuscript review and edit; OMB- Study design, manuscript review and edit; NW- Study design, data collection and manuscript edit; MA-Study design, Data collection, manuscript review and edit; DA- Data collection, manuscript review and edit; TI- Data collection, manuscript review and edit; SA- Data collection, manuscript review and edit; DA- Data collection, manuscript review and edit; JO- Data collection, manuscript review and edit; OA- Data collection, manuscript review and edit; OSA- Data collection, manuscript review and edit; ODA- Data analysis and review, manuscript review and finalization; BA- Data analysis, manuscript review and finalization; BA-K- Data analysis, manuscript review and finalization; OO- Study design, data collection and review, manuscript review and edit; COO-Study concept, study design, data review, manuscript review, edit and finalization. The author(s) read and approved the final manuscript.

\section{Funding}

The randomized control trial was funded by the United Nations Foundation through Global Alliance for Clean Cookstoves (Grant \# UNF12-378) and supported by Richard and Susan Kiphart, Project Gaia and Shell Exploratory Company.

\section{Availability of data and materials}

The datasets generated and analyzed during the current study are not publicly available because they are small data generated from the cohort but are available from the corresponding author on reasonable request.

\section{Declarations}

Ethics approval and consent to participate

The study protocol was explained to the participants in detail and their written informed consents were obtained at the time of recruitment. The work has been carried out in accordance with the Code of Ethics of the World Medical Association (Declaration of Helsinki) and the Institutional Review Boards of the University of Chicago and the University of Ibadan approved the study protocol.

Consent for publication

Not applicable.

Competing interests

The authors declare that they have no competing interests. 


\section{Author details}

'Department of Medicine and Center for Global Health, University of Chicago, Chicago, USA. ${ }^{2}$ Department of Public Health Sciences, University of Chicago, Chicago, USA. ${ }^{3}$ Department of Obstetrics and Gynecology, University of Ibadan, Ibadan, Nigeria. ${ }^{4}$ Pritzker School of Medicine, University of Chicago, Chicago, USA. ${ }^{5}$ Department of Radiology, University of Ibadan, Ibadan, Nigeria. ${ }^{6}$ Healthy Life for All Foundation, House 38, University College Hospital, Ibadan, Nigeria.

Received: 5 January 2021 Accepted: 7 June 2021

Published online: 23 June 2021

\section{References}

1. Barker DJ. The fetal and infant origins of adult disease. BMJ. 1990;301(6761): 1111. https://doi.org/10.1136/bmj.301.6761.1111.

2. Aimakhu CO, Olayemi O. Maternal haematocrit and pregnancy outcome in Nigerian women. West Afr J Med. 2003;22(1):18-21. https://doi.org/10.4314/ wajm.v22i1.27972.

3. Epstein MB, Bates MN, Arora NK, Balakrishnan K, Jack DW, Smith KR. Household fuels, low birth weight, and neonatal death in India: the separate impacts of biomass, kerosene, and coal. Int J Hyg Environ Health. 2013; 216(5):523-32. https://doi.org/10.1016/j.jijheh.2012.12.006.

4. Shah PS, Balkhair T. Air pollution and birth outcomes: a systematic review. Environ Int. 2011;37(2):498-516. https://doi.org/10.1016/j.envint.2010.10.009.

5. Weber E, Adu-Bonsaffoh K, Vermeulen R, Klipstein-Grobusch K, Grobbee DE, Browne $J L$, et al. Household fuel use and adverse pregnancy outcomes in a Ghanaian cohort study. Reprod Health. 2020;17(1):29. https://doi.org/10.11 86/s12978-020-0878-3.

6. Albu AR, Horhoianu IA, Dumitrascu MC, Horhoianu V. Growth assessment in diagnosis of fetal growth restriction. Review J Med Life. 2014;7(2):150-4.

7. ACOG. ACOG Practice Bulletin No. 101: ultrasonography in pregnancy. Obstet Gynecol 2009;113(2 Pt 1):451-461, DOl: https://doi.org/10.1097/AOG. Ob013e31819930b0.

8. Iñiguez C, Ballester F, Estarlich M, Esplugues A, Murcia M, Llop S, et al. Prenatal exposure to traffic-related air pollution and fetal growth in a cohort of pregnant women. Occup Environ Med. 2012;69(10):736-44. https://doi. org/10.1136/oemed-2011-100550.

9. Malmqvist E, Liew Z, Källén K, Rignell-Hydbom A, Rittner R, Rylander L, et al. Fetal growth and air pollution - a study on ultrasound and birth measures. Environ Res. 2017;152:73-80. https://doi.org/10.1016/j.envres.2016.09.017.

10. Smarr MM, Vadillo-Ortega F, Castillo-Castrejon M, O'Neill MS. The use of ultrasound measurements in environmental epidemiological studies of air pollution and fetal growth. Curr Opin Pediatr. 2013;25(2):240-6. https://doi. org/10.1097/MOP.0b013e32835e1e74.

11. van den Hooven EH, Pierik FH, de Kluizenaar Y, Willemsen SP, Hofman A, van Ratingen SW, et al. Air pollution exposure during pregnancy, ultrasound measures of fetal growth, and adverse birth outcomes: a prospective cohort study. Environ Health Perspect. 2012;120(1):150-6. https://doi.org/10.1289/ ehp.1003316.

12. Patelarou E, Kelly FJ. Indoor exposure and adverse birth outcomes related to fetal growth, miscarriage and prematurity-a systematic review. Int J Environ Res Public Health. 2014;11(6):5904-33. https://doi.org/10.3390/ijerph11 0605904.

13. Alexander D, Northcross A, Wilson N, Dutta A, Pandya R, Ibigbami T, et al. Randomized controlled ethanol Cookstove intervention and blood pressure in pregnant Nigerian women. Am J Respir Crit Care Med. 2017;195(12): 1629-39. https://doi.org/10.1164/rccm.201606-11770C.

14. Alexander DA, Northcross A, Karrison T, Morhasson-Bello O, Wilson N, Atalabi OM, et al. Pregnancy outcomes and ethanol cook stove intervention: a randomized-controlled trial in Ibadan. Nigeria Environment international. 2018;111:152-63. https://doi.org/10.1016/j.envint.2017.11.021.

15. Olopade CO, Frank E, Bartlett E, Alexander D, Dutta A, Ibigbami T, et al. Effect of a clean stove intervention on inflammatory biomarkers in pregnant women in Ibadan, Nigeria: a randomized controlled study. Environ Int. 2017; 98:181-90. https://doi.org/10.1016/j.envint.2016.11.004.

16. Hadlock FP, Harrist RB, Carpenter RJ, Deter RL, Park SK. Sonographic estimation of fetal weight. The value of femur length in addition to head and abdomen measurements. Radiology. 1984;150(2):535-40. https://doi. org/10.1148/radiology.150.2.6691115.

17. Northcross A, Shupler M, Alexander D, Olamijulo J, Ibigbami T, Ana G, et al. Sustained usage of bioethanol cookstoves shown in an urban Nigerian city via new SUMs algorithm. Energy Sustainable Dev. 2016;35:35-40. https://doi. org/10.1016/j.esd.2016.05.003.

18. Wu J, Ren C, Delfino RJ, Chung J, Wilhelm M, Ritz B. Association between local traffic-generated air pollution and preeclampsia and preterm delivery in the south coast air basin of California. Environ Health Perspect. 2009; 117(11):1773-9. https://doi.org/10.1289/ehp.0800334.

\section{Publisher's Note}

Springer Nature remains neutral with regard to jurisdictional claims in published maps and institutional affiliations.
Ready to submit your research? Choose BMC and benefit from:

- fast, convenient online submission

- thorough peer review by experienced researchers in your field

- rapid publication on acceptance

- support for research data, including large and complex data types

- gold Open Access which fosters wider collaboration and increased citations

- maximum visibility for your research: over $100 \mathrm{M}$ website views per year

At BMC, research is always in progress.

Learn more biomedcentral.com/submissions 